\title{
Apport du repositionnement du volet osseux sur un lit d'os autogène pour refermer la fenêtre d'accès lors d'un sinus lift avec $A B B M$ et Amoxicilline. Suivi à 4 ans de 83 cas
}

\section{Sage P01, Offerle JM22, Keller P1,2}

1. Cabinet de Chirurgie Orale - Université Louis Pasteur - Strasbourg

2. Hôpitaux Universitaires de Strasbourg (HUS)

\section{Objectif}

L'objectif de ce travail est de décrire les bénéfices du repositionnement du volet osseux sur un lit d'os autogène particulé positionné au dessus de l'ABBM (Anorganic Bovine Bone Minéral, Bio-Oss, Geistlich) afin de poser les implants 3 mois après l'intervention. Nous allons également décrire l'intérêt d'utiliser une solution d'amoxicilline avec du serum physiologique afin de préparer le Bio-oss.

\section{Matériel et méthode}

En 2012, 83 sinus lift ont été réalisés. 15 implants (XiVE, DENTSPLY Implant) ont été placés simultanément au sinus lift et 92 implants ont été mis en place 3 mois après l'intervention. Le volet osseux a été prélevé et préservé dans un milieux aqueux constitué de serum physiologique associé à de l'amoxicilline (si le patient ne présente aucune allergie à la pénicilline). Le volet a été repositionné sur un lit d'os particulé placé sur le matériau de comblement (Bio-Oss, Geistlich), ce qui a permis la stabilisation du caillot sanguin. Le Bio-Oss a été également préparé dans une solution de serum physiologique et amoxicilline. Une radiographie de contrôle a été réalisée 4 ans après la mise en place des implants.

\section{Résultats}

Les 107 implants XiVE (DENTSPLY Implant) sont ostéo intégrés lors du second temps chirurgical. Une perforation est survenue lors du prélèvement d'un volet osseux. Sept perforations ont eu lieu lors du décollement de la membrane sinusienne. Toute les perforations ont été suturées, et une membrane de collagène (PANGEN, Urgo) a été mise en place en complément de la suture. Aucune infection n'a été relevée. Une bonne cicatrisation a été observée à la réouverture. Quatre ans après la mise en place des implants, l'OPT de contrôle montre une bonne stabilité de la greffe.

\section{Discussion}

Les études s'accordent à dire qu'il faut refermer la fenêtre sinusienne. Utiliser une membrane diminue la vitesse de cicatrisation du mur osseux vestibulaire, ce qui repousse la mise en place des implants à 6 mois après le sinus lift (Zinner ID, 1996). De plus, le risque infectieux augmente en cas d'utilisation d'une membrane. Le taux d'échecs des sinus lift dans la littérature varie entre 1,8\% et 14,5\% (Esposito 2010).

\section{Conclusion}

L'os autogène particulé placé sur l'ABBM (Bio-oss, Geistlich) permet de repositionner et de stabiliser le volet osseux et le caillot sanguin. Ceci semble avoir pour effet d'accélérer la cicatrisation du mur osseux vestibulaire et permet la mise en place des implants 3 mois après la première intervention. D'autre part, l'utilisation de serum physiologique en association avec l'amoxicilline pour préparer le Bio-oss et conserver le volet osseux semble diminuer le taux d'échec décrit dans la littérature.

sage.pierre.olivier@gmail.com

(C) The authors, published by EDP Sciences. This is an Open Access article distributed under the terms of the Creative Commons Attribution License 4.0 (http://creativecommons.org/licenses/by/4.0/). 